\title{
"Meat reducers": meat reduction strategies and attitudes towards meat alternatives in an emerging group
}

\author{
J.C. Penny, J.A. Swift and A.M. Salter \\ Division of Nutritional Sciences, School of Biosciences, University of Nottingham, Loughborough, LE12 5RD, UK
}

Diets which reduce, but not completely exclude red meat, have been found to confer, to an intermediate extent, reductions in the risk of coronary heart disease, type 2 diabetes ${ }^{(1)}$ and lower mortality risk ${ }^{(2)}$ when meat is substituted for other healthy protein sources. Therefore, reducing the amount of meat that the UK population eats in their diet has the potential to substantially reduce disease burden and increase disability-adjusted life years ${ }^{(3)}$ and is potentially a more realistic goal for individuals than complete exclusion. However, messages to promote meat reduction diets must be relevant to their target audiences. This study, therefore, aimed to describe the strategies employed and attitudes of omnivorous participants already purposefully reducing their meat consumption.

Similar to previous studies ${ }^{(4)}$, a comprehensive on-line survey was designed to measure a wide range of socio-demographic, behavioural, and cognitive factors associated with meat reduction - a subset of which were used in the current analysis. Omnivores were considered to be a "meat reducer" if they self-identified and/or stated that they were currently trying to reduce, or had successfully reduced their meat consumption. The sample was recruited by SurveyMonkey ${ }^{\mathrm{TM}}$ and selected to be representative of the UK population according to age and gender. Ethical approval was obtained from the School of Biosciences Research Ethics Committee.

The dataset of 1141 responses was screened, and a list-wise deletion approach was applied to missing data and univariate and multivariate outliers; resulting in a useable dataset of 1041 participants. The majority were omnivores $(\mathrm{n}=976)$, and of these, 39.0 \% were meat reducers $(n=381)$ but only $5.2 \%$ self-identified. $6.2 \%$ were vegetarian, vegan, or pescetarian (VVPs). Meat reducers (MRs) reported significantly higher levels of education than omnivores who weren't trying to reduce their meat intake (nMRs; $\mathrm{p}<.01$ ), but no age- or gender-effects were found between MRs and nMRs. Health concerns were rated most highly as reasons for avoiding meat by respondents overall (52.1\% of MRs and 31.1\% of VVPs). However, MRs were more likely to endorse cost and weight control whereas VVPs were more likely to endorse animal welfare and environmental concerns $(\mathrm{p}<.01)$. Amongst MRs, dinner $($ evening meal) was the most common meal to use meat alternatives $(65.2 \%)$, and $39.2 \%$ of MRs reported regularly replacing a portion $(1 / 4-3 / 4)$ of the meat in a meal. When questioned about meat analogues, meat-free days, and reduced meat portions, nMRs were more likely to be concerned about "getting enough iron and/or protein" and "getting enough energy" than MRs, as well as 4-6 times more ambivalent about changing their diet. Both MRs and nMRs reported that a lack of knowledge about the constitution and level of processing of products were major barriers to both traditional and novel meat alternatives (insects, insect proteins, hybrid products and cultured meats).

The implications of this work for health promotion are that: a significant portion of the population are reducing their meat consumption, but new terms such as 'meat reducer' may not be well understood; salient motivations for omnivores are health and weight control, but messages should emphasize that meat reduction strategies can provide a complete and substantial diet; and transparency in terms of the production of meat alternatives is important to consumers.

This research was undertaken for the first author's Masters of Research dissertation and formed part of larger, multidisciplinary, multiagency project funded by Innovate UK.

1. McEvoy C, Temple N, Woodside J (2012) Public Health Nutr 15(12) 2287-2294.

2. Pan A, Sun Q, Bernstein A, et al. (2012) Arch Internal Med. 172(7) 555-563.

3. Friel S, Dangour A, Gernett T, et al. (2009) Lancet 374(9706) 2016-2025.

4. Schösler H, de Boer J, Boersema J (2012) Appetite 58(1) 39-47. 